\title{
ODD DIMENSIONAL RIEMANNIAN SUBMANIFOLDS ADMITTING THE ALMOST CONTACT METRIC STRUCTURE IN A EUCLIDEAN SPHERE
}

\author{
By
}

\author{
Kazuhiro OKumurA
}

\begin{abstract}
We investigate some odd dimensional Rimannian submanifolds admitting the almost contact metric structure $(\phi, \xi, \eta,\langle\rangle$, of a certain Euclidean sphere from the viewpoint of the weakly $\phi$-invariance of the second fundamental form. The family of such submanifolds contains some homogeneous submanifolds of the ambient sphere. In the latter half of this paper, we caluculate the mean curvature and the length of the derivative of the mean curvature vector of these homogeneous submanifolds.
\end{abstract}

\section{Introduction}

We denote by $\left(M^{2 n-1}, l_{M}\right)$ a real hypersurface $M^{2 n-1}$ of an $n$-dimensional complex projective space $\mathbf{C} P^{n}(c)$ of constant holomorphic sectional curvature $c(>0)$ through an isometric immersion $l_{M}: M \rightarrow \mathbf{C} P^{n}(c)$. In [3], Maeda and Udagawa considered a Riemannian submanifold $\left(M^{2 n-1}, f_{1} \circ l_{M}\right)$ of a certain Euclidean sphere by using the isometric parallel minimal embedding $f_{1}: \mathbf{C} P^{n}(c)$ $\rightarrow S^{n(n+2)-1}((n+1) c /(2 n))$, where $S^{n(n+2)-1}((n+1) c /(2 n))$ is an $(n(n+2)-1)$ dimensional sphere of constant sectional curvature $(n+1) c /(2 n)$. Note that this submanifold $\left(M^{2 n-1}, f_{1} \circ l_{M}\right)$ has an almost contact metric structure $(\phi, \xi, \eta,\langle\rangle$, induced from the Kähler structure $J$ and the standard metric $\langle$,$\rangle of \mathbf{C} P^{n}(c)$.

In this paper, we pay particular attention on the structure tensor $\phi$ of $\left(M^{2 n-1}, f_{1} \circ l_{M}\right)$. Motivated by Maeda and Naitoh's work [2], we define the

2000 Mathematics Subject Classification. Primary 53B25, Secondary 53C40.

Key words and phrases. real hypersurfaces, complex projective spaces, real hypersurfaces of type (A), Hopf hypersurfaces, ruled real hypersurfaces, homogeneous submanifold, strongly $\phi$-invariant, weakly $\phi$-invariant, the first standard minimal embedding, Euclidean spheres, mean curvature vector, length of the mean curvature vector.

Received December 4, 2009.

Revised March 15, 2010. 
notion of weakly $\phi$-invariance of the second fundamental form of the immersion $f_{1} \circ l_{M}$ (for details, see Section 4). By virtue of this point of view we can characterize two interesting examples of Riemannian submanifolds $\left(M^{2 n-1}, f_{1} \circ l_{M}\right)$ of the sphere $S^{n(n+2)-1}((n+1) c /(2 n))$ (Theorem 1). One is a homogeneous submanifold and the other is a non-homogeneous submanifold of this sphere.

In this context, we are interested in a homogeneous submanifold $\left(M^{2 n-1}\right.$, $\left.f_{1} \circ l_{M}\right)$ with weakly $\phi$-invariant shape operator in $S^{n(n+2)-1}((n+1) c /(2 n))$. We remark that this manifold $M^{2 n-1}$ is congruent to a homogeneous real hypersurface of type (A) in $\mathbf{C} P^{n}(c)$, namely $M^{2 n-1}$ is congruent to a tube of radius $r(0<r<\pi / \sqrt{c})$ around a totally geodesic Kähler submanifold $\mathbf{C} P^{\ell}(c)(1 \leqq$ $\ell \leqq n-1)$ of $\mathbf{C} P^{n}(c)$, so that $M^{2 n-1}$ is an orbit of some subgroup of the isometry group $S U(n+1)$ of $\mathbf{C} P^{n}(c)$. We compute the mean curvature and the length of the derivative of the mean curvature vector of this homogeneous submanifold $\left(M^{2 n-1}, f_{1} \circ l_{M}\right)$ in the sphere $S^{n(n+2)-1}((n+1) c /(2 n))$ (Theorem 2).

The author would like to thank Professor S. Maeda for his valuable suggestions and encouragement during the preparation of this paper. The author would also like to thank the referee for valuable comments.

\section{Real Hypersurface of Type (A) in $\mathbf{C} P^{n}(c)$}

Let $M^{2 n-1}$ be a real hypersurface with a unit local vector field $\mathscr{N}$ of $\mathbf{C} P^{n}(c)$ through an isometric immersion $l_{M}$. The Riemannian connections $\nabla^{(1)}$ of $\mathbf{C} P^{n}(c)$ and $\nabla$ of $M$ are related by

$$
\begin{gathered}
\nabla_{X}^{(1)} Y=\nabla_{X} Y+\langle A X, Y\rangle \mathscr{N}, \\
\nabla_{X}^{(1)} \mathscr{N}=-A X
\end{gathered}
$$

for vector fields $X$ and $Y$ tangent to $M$, where $\langle$,$\rangle denotes the induced$ metric from the standard Riemannian metric of $\mathbf{C} P^{n}(c)$ and $A$ is the shape operator of $M$ in $\mathbf{C} P^{n}(c)$. (2.1) is called Gauss's formula, and (2.2) is called Weingarten's formula. It is known that $M$ admits an almost contact metric structure $(\phi, \xi, \eta,\langle\rangle$,$) induced from the Kähler structure J$ of $\mathbf{C} P^{n}(c)$. The characteristic vector field $\xi$ of $M$ is defined as $\xi=-J \mathscr{N}$ and this structure satisfies

$$
\phi^{2}=-I+\eta \otimes \xi, \quad \eta(\xi)=1 \quad \text { and } \quad\langle\phi X, \phi Y\rangle=\langle X, Y\rangle-\eta(X) \eta(Y)
$$


where $I$ denotes the identity map of the tangent bundle $T M$ of $M$. It follows from the fact that $\nabla^{(1)} J=0$ and Equations (2.1) and (2.2) that

$$
\nabla_{X} \xi=\phi A X
$$

Eigenvalues and eigenvectors of the shape operator $A$ are called principal curvatures and principal vectors of $M$ in $\mathbf{C} P^{n}(c)$, respectively. In the following, we denote by $V_{\lambda}$ the eigenspace associated to the principal curvature $\lambda$, namely we set $V_{\lambda}=\{v \in T M \mid A v=\lambda v\}$.

We usually call $M$ a Hopf hypersurface if the characteristic vector $\xi$ is a principal curvature vector at each point of $M$. It is known that every tube of sufficiently small constant radius around each Kähler submanifold of $\mathbf{C} P^{n}(c)$ is a Hopf hypersurface. This fact tells us that the notion of Hopf hypersurface is natural in the theory of real hypersurfaces in $\mathbf{C} P^{n}(c)$ (see [4]).

The following lemma clarifies a fundamental property on Hopf hypersurfaces in $\mathbf{C} P^{n}(c), n \geqq 2$.

Lemma 1. For a Hopf hypersurface $M^{2 n-1}(n \geqq 2)$ with principal curvature $\alpha$ corresponding to the characteristic vector field $\xi$ in $\mathbf{C} P^{n}(c)$, we have the following:

(1) $\alpha$ is locally constant on $M$;

(2) If $X$ is a tangent vector of $M$ perpendicular to $\xi$ with $A X=\lambda X$, then

$$
A \phi X=\frac{\alpha \lambda+(c / 2)}{2 \lambda-\alpha} \phi X
$$

The following real hypersurfaces are so-called real hypersurfaces of type $\left(A_{1}\right)$ and $\left(\mathrm{A}_{2}\right)$, respectively.

$\left(\mathrm{A}_{1}\right)$ A geodesic sphere $G(r)$ of radius $r(0<r<\pi / \sqrt{c})$ in $\mathbf{C} P^{n}(c)$.

$\left(\mathrm{A}_{2}\right)$ A tube of radius $r(0<r<\pi / \sqrt{c})$ around a totally geodesic Kähler submanifold $\mathbf{C} P^{\ell}(c)$ in $\mathbf{C} P^{n}(c)$ with $1 \leqq \ell \leqq n-2$.

In this paper, summing up the real hypersurfaces of type $\left(A_{1}\right)$ and type $\left(A_{2}\right)$, we call them the real hypersurfaces of type (A). The real hypersurfaces of type (A) are known as typical examples of Hopf hypersurfaces. The tangent bundle $T M$ of real hypersurfaces $M$ of type $\left(\mathrm{A}_{1}\right)$ with radius $r(0<r<\pi / \sqrt{c})$ is decomposed as $T M=\{\xi\}_{\mathbf{R}} \oplus V_{\lambda}$ with $\alpha=\sqrt{c} \cot (\sqrt{c} r), \lambda=(\sqrt{c} / 2) \cot (\sqrt{c} r / 2), \operatorname{dim}_{\mathbf{R}} V_{\lambda}=$ $2 n-2$ and $\phi V_{\lambda}=V_{\lambda}$. The tangent bundle $T M$ of real hypersurfaces $M$ of type $\left(\mathrm{A}_{2}\right)$ with radius $r(0<r<\pi / \sqrt{c})$ is decomposed as $T M=\{\xi\}_{\mathbf{R}} \oplus V_{\lambda_{1}} \oplus V_{\lambda_{2}}$ with $\quad \alpha=\sqrt{c} \cot (\sqrt{c} r), \quad \lambda_{1}=(\sqrt{c} / 2) \cot (\sqrt{c} r / 2), \quad \lambda_{2}=-(\sqrt{c} / 2) \tan (\sqrt{c} r / 2)$, $\operatorname{dim}_{\mathbf{R}} V_{\lambda_{1}}=2 n-2 \ell-2, \operatorname{dim}_{\mathbf{R}} V_{\lambda_{2}}=2 \ell$ and $\phi V_{i}=V_{i}(i=1,2)$. 
REMARK 1. A geodesic sphere $G(r)$ of radius $r(0<r<\pi / \sqrt{c})$ in $\mathbf{C} P^{n}(c)$ is congruent to a tube of radius $(\pi / \sqrt{c})-r$ around totally geodesic $\mathbf{C} P^{n-1}(c)$ of $\mathbf{C} P^{n}(c)$. In fact, $\lim _{r \rightarrow \pi / \sqrt{c}} G(r)=\mathbf{C} P^{n-1}(c)$.

For the later use we here prepare the following which is a characterization of the real hypersurfaces of type (A) (see [4]).

Lemma 2. Let $M$ be a real hypersurface in $\mathbf{C} P^{n}(c)(n \geqq 2)$. Then the following conditions are mutually equivalent:

(1) $M$ is locally congruent to a real hypersurface of type (A);

(2) $\phi A=A \phi$;

(3) $\left\langle\left(\nabla_{X} A\right) Y, Z\right\rangle=(c / 4)(-\eta(Y)\langle\phi X, Z\rangle-\eta(Z)\langle\phi X, Y\rangle)$ for arbitrary vectors $X, Y$ and $Z$ on $M$.

\section{Ruled Real Hypersurfaces in $\mathbf{C} P^{n}(c)$}

We recall ruled real hypersurfaces in $\mathbf{C} P^{n}(c)$, which are typical examples of non-Hopf hypersurfaces. A real hypersurface $M$ is called a ruled real hypersurface of $\mathbf{C} P^{n}(c)(n \geqq 2)$ if the holomorphic distribution $T^{0} M$ defined by $T^{0} M=\{X \in$ $T M \mid X \perp \xi\}$ is integrable and each of its maximal integral manifolds is a totally geodesic complex hyperplane $\mathbf{C} P^{n-1}(c)$ of $\mathbf{C} P^{n}(c)$. A ruled real hypersurface is constructed in the following manner. Given an arbitrary regular real curve $\gamma$ in $\mathbf{C} P^{n}(c)$ which is defined on an interval I we have at each point $\gamma(t)(t \in \mathrm{I})$ a totally geodesic complex hyperplane $\mathbf{C} P_{t}^{n-1}(c)$ orthogonal to the plane spanned by $\{\dot{\gamma}(t), J \dot{\gamma}(t)\}$. Then we see that $M=\bigcup_{t \in I} \mathbf{C} P_{t}^{n-1}(c)$ is a ruled real hypersurface in $\mathbf{C} P^{n}(c)$. The following gives a characterization of ruled real hypersurfaces in terms of the shape operator $A$ of $M$ (see [4]).

Lemma 3. For a real hypersurface $M$ in $\mathbf{C} P^{n}(c)(n \geqq 2)$, the following conditions (1), (2) and (3) are mutually equivalent:

(1) $M$ is a ruled real hypersurface;

(2) The shape operator $A$ of $M$ satisfies the following equalities on the open dense subset $M_{1}=\{x \in M \mid v(x) \neq 0\}$ with a unit vector field $U$ orthogonal to $\xi: A \xi=\mu \xi+v U, A U=v \xi, A X=0$ for an arbitrary tangent vector $X$ orthogonal to $\xi$ and $U$, where $\mu, v$ are differentiable functions on $M_{1}$ by $\mu=\langle A \xi, \xi\rangle$ and $v=\|A \xi-\mu \xi\|$

(3) The shape operator $A$ of $M$ satisfies $\langle A v, w\rangle=0$ for arbitrary tangent vectors $v, w \in T_{x} M$ orthogonal to $\xi_{x}$ at each point $x \in M$. 
We treat a ruled real hypersurface locally, because generally this hypersurface has self-intersections and sigularities. When we study ruled real hypersurfaces, we usually omit points where $\xi$ is principal and suppose that $v$ does not vanish everywhere, namely a ruled hypersurface $M$ is usually supposed $M_{1}=M$.

\section{Preliminaries and Statements of Results}

In order to obtain Theorems 1 and 2, we first define $\phi$-invariances of the second fundamental form of a submanifold $\left(M^{2 n-1}, f_{1} \circ l_{M}\right)$ in the sphere $S^{n(n+2)-1}((n+1) c /(2 n))$. Since $\left(M^{2 n-1}, l_{M}\right)$ is a real hypersurface of $\mathbf{C} P^{n}(c)$, the manifold $M^{2 n-1}$ has an almost contact metric structure $(\phi, \xi, \eta,\langle\rangle$,$) from the$ Kähler structure $J$ and the standard metric $\langle$,$\rangle of \mathbf{C} P^{n}(c)$ (see Section 2). Then the second fundamental form $\sigma$ of the immersion $f_{1} \circ l_{M}$ is called strongly $\phi$ invariant if $\sigma$ satisfies $\sigma(\phi X, \phi Y)=\sigma(X, Y)$ for all vectors $X$ and $Y$ on $M$. Also, it is called weakly $\phi$-invariant if $\sigma$ satisfies $\sigma(\phi X, \phi Y)=\sigma(X, Y)$ for all vectors $X$ and $Y$ on $M$ orthogonal to the characteristic vector $\xi$ on $M$.

We next recall fundamental geometric properties of the isometric embedding $f_{1}: \mathbf{C} P^{n}(c) \rightarrow S^{n(n+2)-1}((n+1) c /(2 n))$. The inner product of the first normal space of $f_{1}$ is given by

$$
\begin{aligned}
\left\langle\sigma_{1}(X, Y), \sigma_{1}(Z, W)\right\rangle= & -(c /(2 n))\langle X, Y\rangle\langle Z, W\rangle+(c / 4)(\langle X, W\rangle\langle Y, Z\rangle \\
& +\langle X, Z\rangle\langle Y, W\rangle+\langle J X, W\rangle\langle J Y, Z\rangle \\
& +\langle J X, Z\rangle\langle J Y, W\rangle) .
\end{aligned}
$$

Here, $\sigma_{1}$ denotes the second fundamental form of $f_{1}$. Equation (4.1) shows the following properties of $f_{1}$ :

(i) $f_{1}$ is minimal;

(ii) $\sigma_{1}(J X, J Y)=\sigma_{1}(X, Y)$ for $\forall X, Y \in T \mathbf{C} P^{n}(c)$ (namely, $\sigma_{1}$ is $J$-invariant);

(iii) $\left\|\sigma_{1}(X, X)\right\|=\sqrt{(n-1) c /(2 n)}$ for each unit vector $X$ on $\mathbf{C} P^{n}(c)$ (that is, $f_{1}$ is $\sqrt{(n-1) c /(2 n)}$-isotropic in the sense of O'Neill [5]).

Note that $\sigma_{1}$ is $J$-invariant is equivalent to saying that the second fundamental form $\sigma_{1}$ of our embedding $f_{1}$ is parallel (for example see [2], Proposition 3). The embedding $f_{1}$ is usually called the first standard minimal embedding.

THEOREM 1. (1) There exists no Riemannian submanifold $\left(M^{2 n-1}, f_{1} \circ l_{M}\right)$ in $S^{n(n+2)-1}((n+1) c /(2 n))$ such that the second fundamental form $\sigma$ of the immersion $f_{1} \circ l_{M}$ is strongly $\phi$-invariant. 
(2) Let $\left(M^{2 n-1}, f_{1} \circ l_{M}\right)$ be a Riemannian submanifold in $S^{n(n+2)-1}((n+1) c /$ $(2 n))$ such that the second fundamental form $\sigma$ of the immersion $f_{1} \circ l_{M}$ is weakly $\phi$-invariant. Then the following hold:

2i) If $\left(M^{2 n-1}, l_{M}\right)$ is a Hopf hypersurface in $\mathbf{C} P^{n}(c)$, then $\left(M^{2 n-1}, l_{M}\right)$ is locally a real hypersurface of type (A) in $\mathbf{C} P^{n}(c)$;

2ii) If the holomorphic distribution $T^{0} M$ of $\left(M^{2 n-1}, l_{M}\right)$ is integrable, then $\left(M^{2 n-1}, l_{M}\right)$ is a ruled real hypersurface in $\mathbf{C} P^{n}(c)$.

Proof. Let $A$ be a shape operator of $M$ in $\mathbf{C} P^{n}(c)$. Then, second fundamental form $\sigma$ of the immersion $f_{1} \circ l_{M}$ is given by

$$
\sigma(X, Y)=\langle A X, Y\rangle \mathscr{N}+\sigma_{1}(X, Y)
$$

for all vectors $X$ and $Y$ on $M$.

(1) Suppose that $\sigma$ is strongly $\phi$-invariant. Then, from (4.2) we have

$$
\left\{\begin{array}{l}
\langle A \phi X, \phi Y\rangle=\langle A X, Y\rangle, \\
\sigma_{1}(\phi X, \phi Y)=\sigma_{1}(X, Y)
\end{array}\right.
$$

for all vectors $X$ and $Y$ on $M$. However, the equation $\sigma_{1}(\phi X, \phi Y)=\sigma_{1}(X, Y)$ for $\forall X, Y \in T M$ does not hold. Indeed, setting $X=Y=\xi$, we get $\sigma_{1}(\xi, \xi)=0$, which cotradicts the property (iii) of the immersion $f_{1}$. Therefore $\sigma$ is not strongly $\phi$-invariant.

(2) Since $\sigma$ is weakly $\phi$-invariant, from (4.2) we have

$$
\left\{\begin{array}{c}
\langle A \phi X, \phi Y\rangle=\langle A X, Y\rangle \\
\sigma_{1}(\phi X, \phi Y)=\sigma_{1}(X, Y)
\end{array}\right.
$$

for all vectors $X, Y \in T^{0} M$. On the other hand, using the property (ii) of $f_{1}$, we have

$$
\begin{aligned}
\sigma_{1}(\phi X, \phi Y)= & \sigma_{1}(J X-\langle J X, J \xi\rangle J \xi, J Y-\langle J Y, J \xi\rangle J \xi) \\
= & \sigma_{1}(J X, J Y)-\langle J Y, J \xi\rangle \sigma_{1}(J X, J \xi) \\
& -\langle J X, J \xi\rangle \sigma_{1}(J Y, J \xi)+\langle J X, J \xi\rangle\langle J Y, J \xi\rangle \sigma_{1}(J \xi, J \xi) \\
= & \sigma_{1}(X, Y)-\langle Y, \xi\rangle \sigma_{1}(X, \xi)-\langle X, \xi\rangle \sigma_{1}(Y, \xi) \\
& +\langle X, \xi\rangle\langle Y, \xi\rangle \sigma_{1}(\xi, \xi) . \\
= & \sigma_{1}(X, Y) \text { for all vectors } X, Y \in T^{0} M .
\end{aligned}
$$


The above equation implies that in the case $2 \mathrm{i}$ ) we have only to determine Hopf hypersurfaces $M$ of $\mathbf{C} P^{n}(c)$ having $\langle A \phi X, \phi Y\rangle=\langle A X, Y\rangle$ for all $X, Y \in$ $T^{0} M$. We take an unit vector $X \in T^{0} M$ with $A X=\lambda X$. Then, by weakly $\phi-$ invariance, we see that

$$
\langle A \phi X, \phi X\rangle=\langle A X, X\rangle=\lambda .
$$

Moreover, using the assumption that $M$ is a Hopf hypersurface, we obtain $\phi A \xi=0=A \phi \xi$. Hence, we can see that $\phi A=A \phi$ holds on $T M$, so that by Lemma 2, $M$ is a real hypersurface of type (A). Thus we have proved Statement of $2 \mathrm{i}$ ).

Next, we investigate Statement 2ii). So, we suppose that the holomorphic distribution $T^{0} M$ of $M$ is integrable. This assumpution is equivalent to saying that

$$
\langle(\phi A+A \phi) X, Y\rangle=0 \text { for } \forall X, Y \in T^{0} M .
$$

(See Proposion 5 of Kimura and Maeda [1]).

We next show that our real hypersurface $M$ satsfies the following:

$$
\langle A X, Y\rangle=0 \text { for arbitrary } X, Y \in T^{0} M \text {. }
$$

Indeed,

$$
\begin{aligned}
\langle A X, Y\rangle & =\langle A \phi X, \phi Y\rangle \\
& =-\langle\phi A X, \phi Y\rangle \quad(\text { from }(4.4)) \\
& =\left\langle A X, \phi^{2} Y\right\rangle \quad(\text { from the skew-symmetry of } \phi) \\
& =-\langle A X, Y\rangle=0,
\end{aligned}
$$

so that by (3) of Lemma 3, $M$ is a ruled real hypersurface. Therefore we get Statement 2ii).

THEOREM 2. For a real hypersurface $\left(M, l_{M}\right)$ of type $(\mathrm{A})$ in $\mathbf{C} P^{n}(c)$, namely a tube $\left(M, l_{M}\right)$ of radius $r(0<r<\pi / \sqrt{c})$ around totally geodesic $\mathbf{C} P^{\ell}(c)(1 \leqq \ell \leqq$ $n-1)$ in $\mathbf{C} P^{n}(c)$, we denote by $\mathfrak{h}_{\ell}(r)$ the mean curvature vector of the immersion $f_{1} \circ l_{M}: M \rightarrow S^{n(n+2)-1}((n+1) c /(2 n))$. Then we obtain the following statements (1) and (2).

(1) The mean curvature $H_{\ell}(r):=\left\|\mathfrak{h}_{\ell}(r)\right\|$ is given by

$$
H_{\ell}(r)^{2}=\frac{1}{(2 n-1)^{2}}\left[(\text { Trace } A)^{2}+\frac{(n-1) c}{2 n}\right] .
$$


Here, $A$ is the shape operator of our real hypersurface $\left(M, \imath_{M}\right)$ in $\mathbf{C} P^{n}(c)$ and Trace $A$ is given by

(4.6) Trace $A=(2 n-2 \ell-1) \frac{\sqrt{c}}{2} \cot \left(\frac{\sqrt{c}}{2} r\right)-(2 \ell+1) \frac{\sqrt{c}}{2} \tan \left(\frac{\sqrt{c}}{2} r\right)$.

In this case, when Trace $A=0$, i.e. $\tan ^{2}(\sqrt{c} r / 2)=(2 n-2 \ell-1) /(2 \ell+1)$, $H_{\ell}(r)$ has the minimal value $\sqrt{((n-1) c) / 2 n(2 n-1)^{2}}$ which is independent of $\ell(1 \leqq \ell \leqq n-1)$.

(2) The length of the derivative of the mean curvature vector $\left\|D \mathfrak{h}_{\ell}(r)\right\|$ is given by

$$
\begin{aligned}
\left\|D \mathfrak{h}_{\ell}(r)\right\|^{2}= & \frac{c^{2}}{8(2 n-1)^{2}}\left[( n - \ell - 1 ) \left\{(2 n-2 \ell+1) \cot \left(\frac{\sqrt{c}}{2} r\right)\right.\right. \\
& \left.-(2 \ell+1) \tan \left(\frac{\sqrt{c}}{2} r\right)\right\}^{2}+\ell\left\{(2 n-2 \ell-1) \cot \left(\frac{\sqrt{c}}{2} r\right)\right. \\
& \left.\left.-(2 \ell+3) \tan \left(\frac{\sqrt{c}}{2} r\right)\right\}^{2}\right]
\end{aligned}
$$

where $D$ is the normal connection of the immersion $f_{1} \circ l_{M}$. In particular, $D \mathfrak{h}_{\ell}(r)=0$, namely the immersion $f_{1} \circ l_{M}: M \rightarrow S^{n(n+2)-1}((n+1) c /(2 n))$ has parallel mean curvature vector with respect to the normal connection $D$ if and only if $\ell=n-1$ and $\tan ^{2}(\sqrt{c} r / 2)=(2 n-2 \ell-1) /(2 \ell+3)$. This means that $D \mathfrak{h}_{\ell}(r)=0$ if and only if $M$ is a tube of radius $r=(2 / \sqrt{c}) \tan ^{-1}(1 / \sqrt{2 n+1})$ around totally geodesic $\mathbf{C} P^{n-1}(c)$ in $\mathbf{C} P^{n}(c)$, that is $M$ is a geodesic sphere $G(r)$ of radius $r=$ $(2 / \sqrt{c}) \tan ^{-1}(\sqrt{2 n+1})$ of $\mathbf{C} P^{n}(c)$.

Proof. (1) We take a local field of orthonormal frame $\left\{e_{1}, \ldots, e_{n-1}\right.$, $\left.\phi e_{1}\left(=J e_{1}\right), \ldots \phi e_{n-1}\left(=J e_{n-1}\right), \xi\right\}$ on M. Then, as a matter of course $\left\{e_{1}, \ldots, e_{n-1}\right.$, $\left.J e_{1}, \ldots J e_{n-1}, \xi, J \xi(=\mathscr{N})\right\}$ is a local field of orthonormal frames of $\mathbf{C} P^{n}(c)$ along $M$. It follows from the definition of the mean curvature vector that $\mathfrak{h}_{\ell}(r)$ is expressed as

$$
\mathfrak{h}_{\ell}(r)=\frac{1}{2 n-1}\left[(\text { Trace } A) \mathscr{N}+\sum_{i=1}^{n-1}\left(\sigma_{1}\left(e_{i}, e_{i}\right)+\sigma_{1}\left(J e_{i}, J e_{i}\right)\right)+\sigma_{1}(\xi, \xi)\right],
$$

so that

$$
\mathfrak{h}_{\ell}(r)=\frac{1}{2 n-1}\left[(\text { Trace } A) \mathscr{N}-\sigma_{1}(\xi, \xi)\right]
$$


Here, we have used the property (i) of the immersion $f_{1}$. Hence, it follows from the property (iii) of the immersion $f_{1}$ that we obtain (4.5). We find easily that

$$
\text { Trace } A=(2 n-2 \ell-2) \frac{\sqrt{c}}{2} \cot \left(\frac{\sqrt{c}}{2} r\right)-2 \ell \frac{\sqrt{c}}{2} \tan \left(\frac{\sqrt{c}}{2} r\right)+\sqrt{c} \cot (\sqrt{c} r) \text {. }
$$

This, together with $\sqrt{c} \cot (\sqrt{c} r)=(\sqrt{c} / 2) \cot (\sqrt{c} r / 2)-(\sqrt{c} / 2) \tan (\sqrt{c} r / 2)$, yields (4.6).

(2) We take again a local field of orthonormal frames $\left\{e_{1}, \ldots, e_{2 n-2 l-2}\right.$, $\left.f_{1}, \ldots, f_{2 l}, \xi\right\}$ on $M$ such that $e_{i} \in V_{(\sqrt{c} / 2) \cot (\sqrt{c} r / 2)}(1 \leqq i \leqq 2 n-2 \ell-2)$ and $f_{j} \in$ $V_{-(\sqrt{c} / 2) \tan (\sqrt{c} r / 2)}(1 \leqq j \leqq 2 \ell)$. Then $\left\|D \mathfrak{h}_{\ell}(r)\right\|$ is expressed as

$$
\left\|D \mathfrak{h}_{\ell}(r)\right\|^{2}=\left\|D_{\xi} \mathfrak{h}_{\ell}(r)\right\|^{2}+\sum_{i=1}^{2 n-2 \ell-2}\left\|D_{e_{i}} \mathfrak{h}_{\ell}(r)\right\|^{2}+\sum_{j=1}^{2 \ell}\left\|D_{f_{j}} \mathfrak{h}_{\ell}(r)\right\|^{2} .
$$

We shall calculate $\left\|D_{\xi} \mathfrak{h}_{\ell}(r)\right\|,\left\|D_{e_{i}} \mathfrak{h}_{\ell}(r)\right\|$ and $\left\|D_{f_{j}} \mathfrak{h}_{\ell}(r)\right\|$ one by one. In the following computation, we shall use fundamental equations and terminologies in submanifold theory without explanation. Now we denote by $(*)^{\perp}$ the normal component of the vector $(*)$ along $M$ in $T S^{n(n+2)-1}((n+1) c /(2 n)), \nabla^{(2)}$ the Riemannian connection of $S^{n(n+2)-1}((n+1) c /(2 n)), A^{(1)}$ the shape oparetor of $\mathbf{C} P^{n}(c)$ in $S^{n(n+2)-1}((n+1) c /(2 n))$ and $D^{(1)}$ the normal connection of $\mathbf{C} P^{n}(c)$ in $S^{n(n+2)-1}((n+1) c /(2 n))$.

We have

$$
\begin{aligned}
D_{\xi} \mathscr{N} & =\left(\nabla_{\xi}^{(2)} \mathscr{N}\right)^{\perp}=\left(\nabla_{\xi}^{(1)} \mathscr{N}+\sigma_{1}(\xi, \mathscr{N})\right)^{\perp} \quad(\text { from }(2.1)) \\
& =\left(-A \xi+\sigma_{1}(\xi, J \xi)\right)^{\perp} \quad(\text { from }(2.2)) \\
& \left.=\sigma_{1}(\xi, J \xi)=-\sigma_{1}(J \xi, \xi)=0 \quad \text { (from the property (ii) of } f_{1}\right)
\end{aligned}
$$

and

$$
\begin{aligned}
& D_{\xi}\left(\sigma_{1}(\xi, \xi)\right) \\
& \quad=\left(\nabla_{\xi}^{(2)}\left(\sigma_{1}(\xi, \xi)\right)\right)^{\perp} \\
& \quad=\left(-A_{\sigma_{1}(\xi, \xi)}^{(1)} \xi+D_{\xi}^{(1)}\left(\sigma_{1}(\xi, \xi)\right)\right)^{\perp} \quad \text { (from Weingarten's formula) } \\
& \left.\quad=-\left\langle A_{\sigma_{1}(\xi, \xi)}^{(1)} \xi, J \xi\right\rangle J \xi+2 \sigma_{1}\left(\nabla_{\xi}^{(1)} \xi, \xi\right) \quad \text { (from the parallelism of } \sigma_{1}\right) \\
& \quad=-\left\langle\sigma_{1}(\xi, \xi), \sigma_{1}(\xi, J \xi)\right\rangle J \xi+2 \sigma_{1}\left(\nabla_{\xi}^{(1)} \xi, \xi\right)
\end{aligned}
$$




$$
\begin{aligned}
& \left.=2 \sigma_{1}\left(\nabla_{\xi} \xi+\langle A \xi, \xi\rangle J \xi, \xi\right) \quad \text { (from the property (ii) of } f_{1} \text { and }(2.1)\right) \\
& =2 \sigma_{1}(\phi A \xi, \xi)=0 \quad\left(\text { from }(2.4) \text { and the property (ii) of } f_{1}\right)
\end{aligned}
$$

Hence, we obtain

$$
D_{\xi} \mathfrak{h}_{\ell}(r)=0 .
$$

Next, for $e_{i}(1 \leqq i \leqq 2 n-2 \ell-2)$ we see that

$$
\begin{aligned}
D_{e_{i}} \mathscr{N} & =\left(\nabla_{e_{i}}^{(2)} \mathscr{N}\right)^{\perp}=\left(\nabla_{e_{i}}^{(1)} \mathscr{N}+\sigma_{1}\left(e_{i}, \mathscr{N}\right)\right)^{\perp} \\
& =\left(-A e_{i}+\sigma_{1}\left(e_{i}, J \xi\right)\right)^{\perp}=\sigma_{1}\left(e_{i}, J \xi\right)
\end{aligned}
$$

and

$$
\begin{aligned}
D_{e_{i}}\left(\sigma_{1}(\xi, \xi)\right) & =\left(\nabla_{e_{i}}^{(2)}\left(\sigma_{1}(\xi, \xi)\right)\right)^{\perp} \\
& =\left(-A_{\sigma_{1}(\xi, \xi)}^{(1)} e_{i}+D_{e_{i}}^{(2)}\left(\sigma_{1}(\xi, \xi)\right)\right)^{\perp} \quad(\text { from Weingarten's formula) } \\
& =-\left\langle A_{\sigma_{1}(\xi, \xi)}^{(1)} e_{i}, J \xi\right\rangle J+2 \sigma_{1}\left(\nabla_{e_{i}}^{(1)} \xi, \xi\right) \quad\left(\text { from the parallelism of } \sigma_{1}\right) \\
& =-\left\langle\sigma_{1}(\xi, \xi), \sigma_{1}\left(e_{i}, J \xi\right)\right\rangle J \xi+2 \sigma_{1}\left(\nabla_{e_{i}} \xi+\left\langle A e_{i}, \xi\right\rangle J \xi, \xi\right) \quad(\text { from }(2.1)) \\
& =2 \sigma_{1}\left(\nabla_{e_{i}} \xi, \xi\right) \quad\left(\text { from }(4.1) \text { and the property (ii) of } f_{1}\right) \\
& =2 \sigma_{1}\left(\phi A e_{i}, \xi\right) \quad(\text { from }(2.4)) \\
& =\sqrt{c} \cot \left(\frac{\sqrt{c}}{2} r\right) \sigma_{1}\left(\phi e_{i}, \xi\right)=\sqrt{c} \cot \left(\frac{\sqrt{c}}{2} r\right) \sigma_{1}\left(J e_{i}, \xi\right) \\
& =-\sqrt{c} \cot \left(\frac{\sqrt{c}}{2} r\right) \sigma_{1}\left(e_{i}, J \xi\right) .
\end{aligned}
$$

Thus we get

$$
\begin{aligned}
D_{e_{i}} \mathfrak{h}_{\ell}(r)= & \frac{\sqrt{c}}{2(2 n-1)}\left[(2 n-2 \ell+1) \cot \left(\frac{\sqrt{c}}{2} r\right)\right. \\
& \left.-(2 \ell+1) \tan \left(\frac{\sqrt{c}}{2} r\right)\right] \sigma_{1}\left(e_{i}, J \xi\right) .
\end{aligned}
$$

Similarly,

$$
\begin{aligned}
D_{f_{j}} \mathfrak{h}_{\ell}(r)= & \frac{\sqrt{c}}{2(2 n-1)}\left[(2 n-2 \ell-1) \cot \left(\frac{\sqrt{c}}{2} r\right)\right. \\
& \left.-(2 \ell+3) \tan \left(\frac{\sqrt{c}}{2} r\right)\right] \sigma_{1}\left(f_{j}, J \xi\right) .
\end{aligned}
$$


By (4.1) we have

$$
\left\langle\sigma_{1}\left(e_{i}, J \xi\right), \sigma_{1}\left(e_{i}, J \xi\right)\right\rangle=\frac{c}{4} .
$$

Hence, from (4.11), (4.12) and (4.13) we can get

$$
\begin{aligned}
\left\|D_{e_{i}} \mathfrak{h}_{\ell}(r)\right\|^{2}= & \frac{c^{2}}{16(2 n-1)^{2}}\left[(2 n-2 \ell+1) \cot \left(\frac{\sqrt{c}}{2} r\right)\right. \\
& \left.-(2 \ell+1) \tan \left(\frac{\sqrt{c}}{2} r\right)\right]^{2}, \\
\left\|D_{f_{j}} \mathfrak{h}_{\ell}(r)\right\|^{2}= & \frac{c^{2}}{16(2 n-1)^{2}}\left[(2 n-2 \ell-1) \cot \left(\frac{\sqrt{c}}{2} r\right)\right. \\
& \left.-(2 \ell+3) \tan \left(\frac{\sqrt{c}}{2} r\right)\right]^{2} .
\end{aligned}
$$

Thus from (4.9), (4.10), (4.14) and (4.15) we obtain (4.7).

Finally, we shall show that $D \mathfrak{h}_{\ell}(r)=0$ if and only if $\ell=n-1$ and $\tan ^{2}(\sqrt{c} r / 2)=1 /(2 n+1)$. It is obvious the "if" part.

So, we suppose that $D \mathfrak{h}_{\ell}(r)=0$. Since $\ell>0$ and $n-\ell-1 \geqq 0$, from (4.7) and $D \mathfrak{h}_{\ell}(r)=0$ we find the following equations:

$$
(n-\ell-1)\left\{(2 n-2 \ell+1) \cot \left(\frac{\sqrt{c}}{2} r\right)-(2 \ell+1) \tan \left(\frac{\sqrt{c}}{2} r\right)\right\}=0
$$

and

$$
(2 n-2 \ell-1) \cot \left(\frac{\sqrt{c}}{2} r\right)-(2 \ell+3) \tan \left(\frac{\sqrt{c}}{2} r\right)=0 .
$$

So, from (4.17) we have

$$
\tan ^{2}\left(\frac{\sqrt{c}}{2} r\right)=\frac{2 n-2 \ell-1}{2 \ell+3} .
$$

We here remark that in (4.16)

$$
(2 n-2 \ell+1) \cot \left(\frac{\sqrt{c}}{2} r\right)-(2 \ell+1) \tan \left(\frac{\sqrt{c}}{2} r\right) \neq 0 .
$$

In fact we suppose that

$$
(2 n-2 \ell+1) \cot \left(\frac{\sqrt{c}}{2} r\right)-(2 \ell+1) \tan \left(\frac{\sqrt{c}}{2} r\right)=0
$$


which implies that

$$
\tan ^{2}\left(\frac{\sqrt{c}}{2} r\right)=\frac{2 n-2 \ell+1}{2 \ell+1} .
$$

Solving equations (4.18) and (4.19), we find that $n=-1$, which contradicts $n \geqq 2$. Therefore, we get $\ell=n-1$.

REMARK 2. It is known that an isometric immersion $f$ of a Kähler manifold $M$ with Kähler structure $J$ into a sphere has parallel second fundamental form $\sigma$ if and only if $\sigma$ is $J$-invariant, that is $\sigma(J X, J Y)=\sigma(X, Y)$ holds for each vector $X, Y$ on $M$. On the other hand, there exist no submanifolds $\left(M^{2 n-1}, f_{1} \circ l_{M}\right)$ with parallel second fundamental form in the sphere $S^{n(n+2)-1}((n+1) c /(2 n))$. However our homogeneous submanifolds $\left(M^{2 n-1}, f_{1} \circ l_{M}\right)$ stated in Theorem 2 have the weakly $\phi$-invariant second fundamental form.

\section{References}

[ 1 ] M. Kimura and S. Maeda, On real hypersurfaces of a complex projective space, Math. Z. 202 (1989), 299-311.

[2] S. Maeda and H. Naitoh, Real Hypersurfaces with $\phi$-invariant shape operator in a complex projective space, preprint.

[ 3 ] S. Maeda and S. Udagawa, Construction of a certain homogeneous submanifold with nonzero parallel mean curvature vector in Euclidean sphere, preprint.

[4] R. Niebergall and P. J. Ryan, Real hypersurfaces in complex space forms, in: Tight and Taut submanifolds, T. E. Cecil and S. S. Chern (eds.), Cambridge Univ. Press, 1998, 233-305.

[ 5 ] B. O’Neill, Isotropic immersions and Kaehler immersions, Canad. J. Math. 17 (1965), 905-915.

Department of Mathematics

Saga University

1 Honzyo, Saga, 840-8502, Japan

E-mail address: 09632302@edu.cc.saga-u.ac.jp 